\title{
PROJECTIVE MODULES OVER FINITE GROUPS
}

\author{
BY RICHARD G. SWAN ${ }^{1}$
}

Communicated by Irving Kaplansky, August 7, 1959

Serre [5] has recently proved a general theorem about projective modules over commutative rings. This theorem has the following consequence : If $\pi$ is a finite abelian group, any finitely generated projective module over the integral group ring $Z \pi$ is the direct sum of a free module and an ideal of $Z \pi$. The question naturally arises as to whether this result holds for nonabelian groups $\pi$. Serre's methods rely heavily on commutativity and do not seem to generalize to the nonabelian case. However, by using different methods, I have been able to prove the following theorem which generalizes Serre's result even for abelian groups.

THEOREM 1. Let $\pi$ be any finite group. Then any finitely generated projective module over the integral ${ }^{2}$ group ring $Z \pi$ is the direct sum of a free module and an ideal $I$ of $Z \pi$. Furthermore, $I$ can be chosen so that its index in $Z \pi$ (necessarily finite if $I \neq 0$ ) is prime to any given integer.

The main step in the proof is to show that any projective $Z \pi$ module can be imbedded in a free $Z \pi$-module with finite index prime to the order of $\pi$. We then note that any finite $Z \pi$-module of order prime to the order of $\pi$ is weakly projective and hence, by a theorem of $\operatorname{Rim}[4$, Theorem 4.12] has projective dimension 1 . This enables us to split off summands of $Z \pi$-rank 1 from the given projective module. The existence of the required imbedding follows easily from the following theorems.

THEOREM 2. Let $R$ be the ring of p-adic integers. Let $K$ be the quotient field of $R$. Let $P_{1}, P_{2}$ be finitely generated projective modules over the group ring $R \pi$. Suppose that $K \otimes P_{1} \approx K \otimes P_{2}$ over $K \pi$. Then $P_{1} \approx P_{2}$.

The theorem is first proved for abelian $\pi$ by using a projective cover argument. It is then extended to all finite $\pi$ by using a theorem of Artin [1] on induced representations. We also need

${ }^{1}$ Sponsored by the Office of Ordnance Research, U. S. Army under contract DA-11-022-ORD-2911.

${ }^{2}$ For simplicity, I will state the theorems here for the case in which the ground ring is the ring of integers. They actually remain true over any Dedekind ring satisfying suitable additional hypotheses. These hypotheses will always be satisfied by rings of algebraic integers. 
THEOREM 3. Let $Q$ be the field of rational numbers. If $P$ is any finitely generated projective module over $Z \pi$, then $Q \otimes P$ is free over $Q \pi$.

This can be proved by computing the character of the representation of $\pi$ on $Q \otimes P$. Combining Theorems 2 and 3, we get the following result.

CoROLlary. If $P$ is any finitely generated projective module over $Z \pi$, then $P / m P$ is free over $Z_{m} \pi$ for any integer $m \neq 0$.

This gives us immediately the imbedding needed to prove Theorem 1.

The techniques used to prove Theorem 2 yield further results about the projective class group $[4 ; 5] C(Z \pi)$ of $Z \pi$. It follows immediately from Theorem 1 and the Jordan-Minkowski theorem [6] that $C(Z \pi)$ is finite for all finite groups $\pi$. This answers a question of Rim [4]. If $\pi^{\prime} C \pi$, there is an obvious map $i\left(\pi, \pi^{\prime}\right): C(Z \pi) \rightarrow C\left(Z \pi^{\prime}\right)$ obtained by restricting the group of operators on all modules from $\pi$ to $\pi^{\prime}$. Recall that a finite group is called elementary [3] if it is the direct product of a $p$-group and a cyclic group. I will call a finite group hyperelementary if it is a split extension of a $p$-group by a cyclic group (the cyclic group being the kernel).

THEOREM 4. Let $n$ be the order of $\pi$. Let $d$ be the greatest common divisor of $n$ and $\phi(n)$, where $\phi$ is Euler's $\phi$-function. Let $\alpha \in C(Z \pi)$. Then

(1) If $i\left(\pi, \pi^{\prime}\right) \alpha=0$ for all cyclic subgroups $\pi^{\prime}$ of $\pi$, then $n \alpha=0$.

(2) If $i\left(\pi, \pi^{\prime}\right) \alpha=0$ for all elementary subgroups $\pi^{\prime}$ of $\pi$, then $d \alpha=0$.

(3) If $i\left(\pi, \pi^{\prime}\right) \alpha=0$ for all hyperelementary subgroups $\pi^{\prime}$ of $\pi$, then $\alpha=0$.

The proof makes use of the Grothendieck rings $[2 ; 5]$ of the various groups involved. It also uses the theorem of Artin previously mentioned [1], Brauer's theorem on induced representations [3], and the following theorem.

THeOREM 5. Let $\pi$ be any finite group. The character of any rational representation of $\pi$ is an integral linear combination of characters induced from rational representations of hyperelementary subgroups of $\pi$.

The proof is similar to that of Brauer's theorem [3].

\section{REFERENCES}

1. E. Artin, Die Gruppentheoretische Struktur der Discriminanten algebraischer Zahlkörper, J. Reine Angew. Math. vol. 164 (1931) pp. 1-11. 
2. A. Borel and J-P. Serre, Le theorème de Riemann-Roch (d'après A. Grothendieck), Bull. Soc. Math. France vol. 86 (1958) pp. 97-136.

3. R. Brauer and J. Tate, On the characters of finite groups, Ann. of Math. vol. 62 (1955) pp. 1-7.

4. D. S. Rim, Modules over finite groups, Ann. of Math. vol. 69 (1959) pp. 700712.

5. J-P. Serre, Modules projectifs et espaces fibrés a fibre vectorielle, Séminaire Dubreil, Algèbre et Theorie des Nombres, Paris, 1958.

6. H. Zassenhaus, Neuer Beweis der Endlichkeit der Klassenzahl bei unimodularer Aquivalenz endlicher ganzzahliger Substitutionsgruppen, Abh. Math. Sem. Hamburg vol. 12 (1938) pp. 276-288.

The University of Chicago 